\title{
A LOCAL LIMIT THEOREM FOR COEFFICIENTS OF MODIFIED BORWEIN'S METHOD
}

\author{
IGORIS BELOVAS
}

Vilnius University, Lithuania

\begin{abstract}
The paper extends the study of the modified Borwein method for the calculation of the Riemann zeta-function. It presents an alternative perspective on the proof of a local limit theorem for coefficients of the method. The new approach is based on the connection with the limit theorem applied to asymptotic enumeration.
\end{abstract}

\section{INTRODUCTION}

In [1] we introduced a modification of Borwein's method for the calculation of the Riemann zeta-function and proposed an asymptotic expression for the coefficients of the method. The asymptotic modification of the algorithm proved to be more than three times faster than the original one ([1]). Borwein's method for calculating Riemann zeta-function is based on the alternating series convergence ([4]). It applies to complex numbers $s=\sigma+i t$ with $\sigma \geqslant 1 / 2$.

Let

$$
d_{n k}=n \sum_{i=0}^{k} \frac{(n+i-1) ! 4^{i}}{(n-i) !(2 i) !}, \quad n \in \mathbb{N}, \quad 0 \leqslant k \leqslant n,
$$

then the Riemann zeta-function

$$
\zeta(s)=\frac{1}{d_{n n}\left(1-2^{1-s}\right)} \sum_{k=0}^{n-1} \frac{(-1)^{k}\left(d_{n n}-d_{n k}\right)}{(k+1)^{s}}+\gamma_{n}(s),
$$

2010 Mathematics Subject Classification. 05A16, $11 \mathrm{M} 99$.

Key words and phrases. Local limit theorem, asymptotic enumeration, asymptotic normality. 
where

$$
\left|\gamma_{n}(s)\right| \leqslant \frac{3}{(3+\sqrt{8})^{n}} \frac{(1+2|t|) e^{\frac{\pi|t|}{2}}}{\left|1-2^{1-s}\right|} .
$$

It is challenging to compute coefficients $d_{n k}$ for large $n$ directly (because of factorials in the definition). Therefore we have introduced a modification of the method. Let $c_{n k}=1-d_{n k} / d_{n n}, \quad 0 \leqslant k \leqslant n-1$. Now

$$
\zeta(s)=\sum_{k=0}^{n-1} \frac{(-1)^{k} c_{n k}}{(k+1)^{s}}+\gamma_{n}(s) .
$$

Let

$$
u_{n k}=n \frac{(n+k-1) ! 4^{k}}{(n-k) !(2 k) !}, \quad n \in \mathbb{N}, \quad 0 \leqslant k \leqslant n .
$$

Now we can calculate $d_{n k}$ recurrently, i.e. $d_{n k}=d_{n, k-1}+u_{n k}, d_{n 0}=1$, and

$$
c_{n k}=1-\sum_{i=0}^{k} a_{n i}
$$

where

$$
a_{n k}=\frac{u_{n k}}{\sum_{i=0}^{n} u_{n i}}
$$

In [1] we proved a local limit theorem for coefficients of modified Borwein's method. Note that throughout the paper, all limits, whenever unspecified, will be taken as $n \rightarrow \infty$.

Theorem 1.1 (I. Belovas, L. Sakalauskas [1]). Let $\mu_{n}=\frac{n}{\sqrt{2}}, \sigma_{n}=\frac{\sqrt{n}}{2 \sqrt[4]{2}}$. Numbers $a_{n k}$ satisfy a local limit theorem

$$
\lim _{n \rightarrow \infty} \sup _{k}\left|a_{n k}-\varphi_{\mu_{n}, \sigma_{n}}(k)\right|=0,
$$

where $\varphi_{\mu, \sigma}(x)$ is the probability density function of the normal distribution with the mean $\mu$ and the standard deviation $\sigma$.

The theorem was proved in a "straightforward" way, using Stirling's formula. However, alternative perspective reveals the connection with combinatorial numbers and calls for application of the results of asymptotic enumeration theory ([6]). We will use a general local limit theorem by E. A. Bender, based on the nature of the generating function $\sum u_{n k} z^{n} w^{k}$.

Theorem 1.2 (E. A. Bender [3]). Let $f(z, w)$ have a power series expansion

$$
f(z, w)=\sum_{n, k \geqslant 0} u_{n k} z^{n} w^{k}
$$


with non-negative coefficients and let $a<b$ be real numbers. Define

$$
R(\varepsilon)=\{z: a \leqslant \Re z \leqslant b, \quad|\Im z| \leqslant \varepsilon\} .
$$

Suppose there exists $\varepsilon>0, \delta>0$, a non-negative integer $m$, and functions $A(s), r(s)$ such that

(i) an $A(s)$ is continuous and non-zero for $s \in R(\varepsilon)$,

(ii) an $r(s)$ is non-zero and has a bounded third derivative for $s \in R(\varepsilon)$,

(iii) for $s \in R(\varepsilon)$ and $|z| \leqslant|r(s)|(1+\delta)$ function

$$
\left(1-\frac{z}{r(s)}\right)^{m} f\left(z, e^{s}\right)-\frac{A(s)}{1-z / r(s)}
$$

is analytic and bounded,

(iv) $\left(r^{\prime}(\alpha) / r(\alpha)\right)^{2}-r^{\prime \prime}(\alpha) / r(\alpha) \neq 0$ for $a \leqslant \alpha \leqslant b$,

(v) $f\left(z, e^{s}\right)$ is analytic and bounded for

$$
|z| \leqslant|r(\Re s)|(1+\delta), \quad \varepsilon \leqslant|\Im s| \leqslant \pi .
$$

Then we have

$$
u_{n k} \sim \frac{n^{m} e^{-\alpha k} A(\alpha)}{m ! r^{n}(\alpha) \vartheta_{\alpha} \sqrt{2 \pi n}}
$$

uniformly for $a \leqslant \alpha \leqslant b$, where

$$
\frac{k}{n}=-\frac{r^{\prime}(\alpha)}{r(\alpha)}, \quad \vartheta_{\alpha}=\left(\frac{k}{n}\right)^{2}-\frac{r^{\prime \prime}(\alpha)}{r(\alpha)} .
$$

\section{LOCAL LIMIT THEOREM FOR THE COEFFICIENTS $u_{n k}$}

First, we prove an auxiliary lemma, identifing the generating function (1.3) of coefficients $u_{n k}$ (1.1).

LEMma 2.1. Suppose that

$$
u_{n k}= \begin{cases}1 & n=k=0 \\ 0 & k>n \\ n \frac{(n+k-1) ! 4^{k}}{(n-k) !(2 k) !} & \text { otherwise }\end{cases}
$$

then the generating function

$$
\sum_{n, k \geqslant 0} u_{n k} x^{n} y^{k}=\frac{1}{2}\left(1+\frac{1}{2 x^{-1} \Theta(y)-1}-\frac{1}{2 x \Theta(y)-1}\right) .
$$

Here

$$
\Theta(y)=y+\sqrt{y+y^{2}}+1 / 2 .
$$


Proof. By definition (2.1), we have the recurrent expression

$$
u_{n k}=u_{n, k-1} \frac{4(n+k-1)(n-k+1)}{(2 k-1)(2 k)} .
$$

Let us consider the generating function (2.2),

$$
f(x, y)=\sum_{n=0}^{\infty} \sum_{k=0}^{\infty} u_{n k} x^{n} y^{k} .
$$

Taking into account that $u_{n 0}=1$ and (2.3), we obtain the expresion

$$
\begin{aligned}
f(x, y) & =\sum_{n=0}^{\infty} u_{n 0} x^{n}+\sum_{n=0}^{\infty} \sum_{k=1}^{\infty} u_{n, k-1} \frac{4(n+k-1)(n-k+1)}{(2 k-1)(2 k)} x^{n} y^{k} \\
& =\frac{1}{1-x}+4 \sum_{n=0}^{\infty} \sum_{k=0}^{\infty} u_{n, k} \frac{(n+k)(n-k)}{(2 k+1)(2 k+2)} x^{n} y^{k+1}
\end{aligned}
$$

yielding the integral equation

$$
\begin{aligned}
f(x, y)= & \frac{1}{1-x}-y f(x, y)+\frac{3}{2} \int_{0}^{y} f(x, t) d t+ \\
& +\int_{0}^{\sqrt{y}} \int_{0}^{u} 4 x f_{x}\left(x, t^{2}\right)+4 x^{2} f_{x x}\left(x, t^{2}\right)-f\left(x, t^{2}\right) d t d u .
\end{aligned}
$$

It gives us the linear partial differential equation of the second order,

$$
x^{2} f_{x x}-\left(y+y^{2}\right) f_{y y}+x f_{x}-(1 / 2+y) f_{y}=0 .
$$

Note that, in view of (2.4), we have initial conditions

$$
f(x, 0)=\frac{1}{1-x}, \quad f_{y}(x, 0)=0 .
$$

Solving the equation (e.g., by the method of characteristics), we obtain

$$
f(x, y)=\frac{1}{2}\left(1+\frac{1}{2 x^{-1} \Theta(y)-1}-\frac{1}{2 x \Theta(y)-1}\right),
$$

which yields us the statement of the lemma.

Now we can proceed with the local limit theorem for coefficients $u_{n k}(2.1)$.

THEOREM 2.2. Let

$$
\mu_{n}=\frac{n}{\sqrt{2}}, \sigma_{n}^{2}=\frac{n \sqrt{2}}{8}
$$

then for all $k$, such that

$$
\left|k-\mu_{n}\right|=o\left(\sigma_{n}^{4 / 3}\right),
$$

we have

$$
u_{n k} \sim \frac{(1+\sqrt{2})^{2 n}}{2 \sqrt{2 \pi} \sigma_{n}} \exp \left(-\frac{\left(k-\mu_{n}\right)^{2}}{2 \sigma_{n}^{2}}\right)
$$


Proof. By Lemma 2.1, the generating function

$$
\begin{aligned}
f\left(z, e^{s}\right) & =\frac{1}{2}\left(1+\frac{1}{2 z^{-1} \Theta(s)-1}-\frac{1}{2 z \Theta(s)-1}\right) \\
& =\frac{4 \Theta^{2}(s)-4 z^{-1} \Theta(s)+1}{2\left(2 z^{-1} \Theta(s)-1\right)(2 z \Theta(s)-1)},
\end{aligned}
$$

where we write $\Theta(s)$ in place of $\Theta\left(e^{s}\right)$.

Let $r(s)$ (cf. Theorem 1.2) be a root of the function

$$
h\left(z, e^{s}\right)=\left(2 z^{-1} \Theta(s)-1\right)(2 z \Theta(s)-1) .
$$

This function has two roots, $z_{1}=2 \Theta(s)$ and $z_{2}=(2 \Theta(s))^{-1}$. Let us denote

$$
r_{1}(s)=2 \Theta(s), \quad r_{2}(s)=\frac{1}{2 \Theta(s)} .
$$

Calculating derivatives, we obtain

$$
\frac{r_{1}^{\prime}(0)}{r_{1}(0)}=\frac{1}{\sqrt{2}}>0, \quad \frac{r_{2}^{\prime}(0)}{r_{2}(0)}=-\frac{1}{\sqrt{2}}<0 .
$$

By Bender $\left(\left[3\right.\right.$, Theorem 1]), the mean $\mu_{n}=n \mu$ and $\mu=-r^{\prime}(0) / r(0)$. Note that by definitions (1.1)-(1.2), numbers $u_{n k}$ and $a_{n k}$ are positive. Thus, to obtain positive $\mu$, we choose the root $r_{2}(s)$, corresponding the negative ratio. Hence, by (2.7), we have

$$
r(s)=r_{2}(s)=\frac{1}{2 \Theta(s)}=\frac{1}{2\left(e^{s}+\sqrt{e^{s}+e^{2 s}}+1 / 2\right)} .
$$

Thus,

$$
\frac{r^{\prime}(s)}{r(s)}=-\sqrt{\frac{e^{s}}{e^{s}+1}}, \quad \frac{r^{\prime}(0)}{r(0)}=-\frac{1}{\sqrt{2}},
$$

and

$$
\frac{r^{\prime \prime}(s)}{r(s)}=\frac{e^{s}}{e^{s}+1}-\frac{1}{2} \sqrt{\frac{e^{s}}{\left(e^{s}+1\right)^{3}}}, \quad \frac{r^{\prime \prime}(0)}{r(0)}=\frac{1}{2}-\frac{\sqrt{2}}{8} .
$$

Next, consider the function $A(s)$ (cf. (1.4) of Theorem 1.2) as the limit

$$
A(s)=\lim _{z \rightarrow r(s)} f\left(z, e^{s}\right)\left(1-\frac{z}{r(s)}\right)^{m+1} .
$$

Here $m+1$ is the order of the pole. Note that, if the pole is simple, then $m=0$. Calculating $A(s)$ we obtain

$$
\begin{aligned}
A(s) & =\lim _{z \rightarrow r(s)} \frac{1}{2}\left(1+\frac{1}{2 z^{-1} \Theta(s)-1}-\frac{1}{2 z \Theta(s)-1}\right)\left(1-\frac{z}{r(s)}\right) \\
& =\lim _{z \rightarrow r(s)} \frac{1}{2}\left(1+\frac{1}{z^{-1} r^{-1}(s)-1}-\frac{1}{z r^{-1}(s)-1}\right)\left(1-\frac{z}{r(s)}\right)=\frac{1}{2} .
\end{aligned}
$$


The function (1.4)

is analytic and bounded for

$$
\left(1-\frac{z}{r(s)}\right)^{m} f\left(z, e^{s}\right)-\frac{A(s)}{1-z / r(s)}=\frac{\Theta(s)}{2 \Theta(s)-z}
$$

$$
|s|<\varepsilon, \quad|z|<|r(0)|+\delta=3-2 \sqrt{2}+\delta .
$$

Thus, conditions (i)-(iii) and (v) of Theorem 1.2 are satisfied. To verify the condition (iv), we must calculate the expression $\left(r^{\prime}(\alpha) / r(\alpha)\right)^{2}-r^{\prime \prime}(\alpha) / r(\alpha)$. By (2.9) and (2.10) we have

$$
\left(\frac{r^{\prime}(\alpha)}{r(\alpha)}\right)^{2}-\frac{r^{\prime \prime}(\alpha)}{r(\alpha)}=\frac{1}{2} \sqrt{\frac{e^{\alpha}}{\left(e^{\alpha}+1\right)^{3}}} \neq 0 .
$$

We obtain the parameter $\alpha$ by solving the equation

$$
\frac{k}{n}=-\frac{r^{\prime}(\alpha)}{r(\alpha)} \text {. }
$$

Using (2.9) we get

$$
\frac{k}{n}=\frac{1}{\sqrt{1+e^{-\alpha}}}
$$

Hence,

Next (cf. (1.6) and (2.8)),

$$
e^{\alpha}=\frac{k^{2}}{n^{2}-k^{2}}
$$

$$
\begin{aligned}
& \vartheta_{\alpha}^{2}=\frac{1}{2} \sqrt{\frac{e^{\alpha}}{\left(e^{\alpha}+1\right)^{3}}}=\frac{1}{2 e^{\alpha}}\left(\frac{e^{\alpha}}{e^{\alpha}+1}\right)^{3 / 2}, \\
& r^{n}(\alpha)=\left(2\left(e^{\alpha}+\sqrt{e^{\alpha}+e^{2 \alpha}}+1 / 2\right)\right)^{-n} .
\end{aligned}
$$

Now we can calculate (1.5) of Theorem 1.2,

$$
\begin{aligned}
& u_{n k} \sim \frac{e^{-\alpha k} \frac{1}{2}}{r^{n}(\alpha) \vartheta_{\alpha} \sqrt{2 \pi n}} \\
& =\frac{\left(2\left(e^{\alpha}+\sqrt{e^{\alpha}+e^{2 \alpha}}+1 / 2\right)\right)^{n}}{2 \sqrt{\pi n} e^{\alpha(k-1 / 2)}\left(\frac{e^{\alpha}}{e^{\alpha}+1}\right)^{3 / 4}}=\frac{\left(\frac{n+k}{n-k}\right)^{n}\left(\frac{k}{n}\right)^{-3 / 2}}{2 \sqrt{\pi n}\left(\frac{k^{2}}{n^{2}-k^{2}}\right)^{k-1 / 2}} \\
& =\frac{(1+\sqrt{2})^{2 n}}{2 \sqrt{2 \pi} \sigma_{n}} \frac{\sqrt[4]{2}}{2} \frac{\left(\frac{1+\frac{k}{n}}{1-\frac{k}{n}}\right)^{n}}{(1+\sqrt{2})^{2 n}} \frac{\left(\left(1-\frac{k}{n}\right)\left(1+\frac{k}{n}\right)\right)^{k-1 / 2}}{\left(\frac{k}{n}\right)^{2 k+1 / 2}} \\
& =\frac{(1+\sqrt{2})^{2 n}}{2 \sqrt{2 \pi} \sigma_{n}} \underbrace{\frac{\sqrt[4]{2} / 2}{\sqrt{\left(1-\frac{k}{n}\right)\left(1+\frac{k}{n}\right) \frac{k}{n}}}}_{=\theta_{n k}} \underbrace{\frac{\left(1+\frac{k}{n}\right)^{n+k}\left(1-\frac{k}{n}\right)^{-n+k}}{(1+\sqrt{2})^{2 n}\left(\frac{k}{n}\right)^{2 k}}}_{=\delta_{n k}} .
\end{aligned}
$$


Note that by (2.5) and (2.6), we have

$$
\left|\frac{k}{n}-\frac{1}{\sqrt{2}}\right|=o\left(\frac{1}{\sqrt[3]{n}}\right)
$$

hence $k / n \rightarrow 1 / \sqrt{2}$, while $n \rightarrow \infty$. Thus, $\theta_{n k} \rightarrow 1$.

Let us denote

$$
x=\frac{k-\mu_{n}}{\sigma_{n}}
$$

By (2.5), we have

$$
\frac{k}{n}=\frac{1}{\sqrt{2}}+\frac{x}{2 \sqrt[4]{2} \sqrt{n}}
$$

and by (2.12), we have

$$
|x|=o(\sqrt[6]{n})
$$

Calculating the logarithm of $\delta_{n k}(2.11)$, we get

$$
\begin{aligned}
\log \delta_{n k} & =-2 n \log (1+\sqrt{2})-\left(n \sqrt{2}+\frac{x \sqrt{n}}{\sqrt[4]{2}}\right) \log \left(\frac{1}{\sqrt{2}}+\frac{x}{2 \sqrt[4]{2} \sqrt{n}}\right) \\
+ & \left(n+\frac{n}{\sqrt{2}}+\frac{x \sqrt{n}}{2 \sqrt[4]{2}}\right) \log \left(1+\frac{1}{\sqrt{2}}+\frac{x}{2 \sqrt[4]{2} \sqrt{n}}\right) \\
+ & \left(-n+\frac{n}{\sqrt{2}}+\frac{x \sqrt{n}}{2 \sqrt[4]{2}}\right) \log \left(1-\frac{1}{\sqrt{2}}-\frac{x}{2 \sqrt[4]{2} \sqrt{n}}\right) \\
= & -2 n \log (1+\sqrt{2}) \\
& -\left(n \sqrt{2}+\frac{x \sqrt{n}}{\sqrt[4]{2}}\right)\left(\log \frac{1}{\sqrt{2}}+\log \left(1+\frac{x}{\sqrt{2} \sqrt[4]{2} \sqrt{n}}\right)\right) \\
+ & \left(\frac{1+\sqrt{2}}{\sqrt{2}} n+\frac{x \sqrt{n}}{2 \sqrt[4]{2}}\right)\left(\log \frac{\sqrt{2}+1}{\sqrt{2}}+\log \left(1+\frac{x(\sqrt{2}-1)}{\sqrt{2} \sqrt[4]{2} \sqrt{n}}\right)\right) \\
+ & \left(\frac{1-\sqrt{2}}{\sqrt{2}} n+\frac{x \sqrt{n}}{2 \sqrt[4]{2}}\right)\left(\log \frac{\sqrt{2}-1}{\sqrt{2}}+\log \left(1-\frac{x(\sqrt{2}+1)}{\sqrt{2} \sqrt[4]{2} \sqrt{n}}\right)\right) .
\end{aligned}
$$


Using Taylor series expansions for logarithms, we obtain for large enough $n$,

$$
\begin{aligned}
\log \delta_{n k} & =-2 n \log (1+\sqrt{2})+\left(n \sqrt{2}+\frac{x \sqrt{n}}{\sqrt[4]{2}}\right)\left(\frac{1}{2} \log 2-\right. \\
& \left.-\frac{x}{\sqrt{2} \sqrt[4]{2} \sqrt{n}}+\frac{x^{2}}{4 \sqrt{2} n}+O\left(\frac{x^{3}}{n \sqrt{n}}\right)\right)+\left(\frac{1+\sqrt{2}}{\sqrt{2}} n+\right. \\
& \left.+\frac{x \sqrt{n}}{2 \sqrt[4]{2}}\right)\left(\log \frac{\sqrt{2}+1}{\sqrt{2}}+\frac{x(\sqrt{2}-1)}{\sqrt{2} \sqrt[4]{2} \sqrt{n}}-\frac{x^{2}(\sqrt{2}-1)^{2}}{4 \sqrt{2} n}+\right. \\
& \left.+O\left(\frac{x^{3}}{n \sqrt{n}}\right)\right)+\left(\frac{1-\sqrt{2}}{\sqrt{2}} n+\frac{x \sqrt{n}}{2 \sqrt[4]{2}}\right)\left(\log \frac{\sqrt{2}-1}{\sqrt{2}}-\right. \\
& \left.-\frac{x(\sqrt{2}+1)}{\sqrt{2} \sqrt[4]{2} \sqrt{n}}-\frac{x^{2}(\sqrt{2}+1)^{2}}{4 \sqrt{2} n}+O\left(\frac{x^{3}}{n \sqrt{n}}\right)\right)
\end{aligned}
$$

By multiplying factors and combining like terms, we obtain

$$
\log \delta_{n k}=-\frac{x^{2}}{2}+O\left(\frac{x^{3}}{\sqrt{n}}\right),
$$

which, combined with (2.11) and (2.13), yields us the statement of the theorem.

REMARK 2.3. Theorem 2.2 yields us the asymptotic equivalence

$$
\sum_{k=0}^{n} u_{n k} \sim \frac{1}{2}(1+\sqrt{2})^{2 n}
$$

(cf. [1, Lemma 2.1]).

REMARK 2.4. A central limit theorem for the coefficients of modified Borwein's method can be proved analogically, using Bender's central limit theorem applied to asymptotic enumeration (Theorem 1, [2, 3]. However, the approach, based on Hwang's limit theorem ([5]), yields stronger result, enabling us to evaluate the rate of convergence to normal distribution (cf. [1, Theorem 3.1]).

\section{ACKNOWLEDGEMENTS.}

The author would like to thank the anonymous reviewer for careful reading of the manuscript and providing constructive comments and suggestions, which have helped him to improve the quality of the paper.

\section{REFERENCES}

[1] I. Belovas, L. Sakalauskas, Limit theorems for the coefficients of the modified Borwein method for the calculation of the Riemann zeta-function values, Colloq. Math. 151 (2018), 217-227.

[2] I. Belovas, A central limit theorem for coefficients of the modified Borwein method for the calculation of the Riemann zeta-function, Lith. Math. J. 59 (2019), 17-23. 
[3] E. A. Bender, Central and local limit theorems applied to asymptotic enumeration, J. Combin. Theory Ser. A. 15 (1973), 91-111.

[4] P. Borwein, An efficient algorithm for the Riemann Zeta function, in: Constructive, Experimental, and Nonlinear Analysis (Limoges, 1999), CRC, Boca Raton, 2000, 29-34.

[5] H.-K. Hwang, On convergence rates in the central limit theorems for combinatorial structures European J. Combin., 19 (1998), 329-343.

[6] A. M. Odlyzko, Asymptotic enumeration methods, in: Handbook of Combinatorics, vol. 2, Elsevier Sci. B. V., Amsterdam, 1995, 1063-1229.

I. Belovas

Vilnius University

Institute of Data Science and Digital Technologies

Akademijos st. 4, 08412 Vilnius

Lithuania

and

Vilnius Gediminas Technical University

10223 Vilnius

Lithuania

E-mail: Igoris.Belovas@mii.vu.lt

Received: 22.11 .2018$.

Revised: 16.1.2019. 